\title{
FORMAÇÃO DOCENTE PARA AS ESCOLAS CIDADÃS DA PARAÍBA: CONTRIBUIÇÕES DO PROGRAMA GIRA MUNDO FINLÂNDIA
}

\author{
TEACHER TRAINING FOR CITIZEN SCHOOLS IN PARAÍBA: GIRA MUNDO \\ FINLAND CONTRIBUTIONS
}

\author{
I. M. A. COTRIM GUIMARÃES ${ }^{1, *}$, A. F. D'ANDREA ${ }^{2}$ e J. R. OUVERNEY KING ${ }^{3}$ \\ 1 Instituto Federal do Norte de Minas Gerais, Campus Januária, Brasil. \\ 2 Instituto Federal da Paraíba - Campus João Pessoa, Brasil. \\ ${ }^{3}$ Instituto Federal da Paraíba - Campus Cabedelo, Brasil.
}

\section{ARTICLE INFO}

Article history:

Received 2018-07-02

Accepted 2018-08-17

Available online 2018-08-31

\section{*Autor correspondente:}

E-mail: izacotrim2014@gmail.com
Palavras-chave: Formação de professores. Políticas públicas. Educação profissional. Inovação em educação. Protagonismo juvenil.

Keywords: Teacher training. Public policies. Vocational education. Innovation in education. Youth protagonism.

RESUMO. O Programa Gira Mundo Finlândia (GMF) é uma iniciativa do Governo da Paraíba, por meio da Secretaria de Estado da Educação, e tem como objetivo a capacitação de servidores efetivos da Rede Estadual de Ensino em Universidades de Ciências Aplicadas da Finlândia. Com um período de imersão entre quatro a oito semanas na Finlândia, professores e gestores visitaram escolas, organizações e vivenciaram o sistema educacional finlandês. No retorno à Paraíba, desenvolveram um projeto de aprimoramento das práticas pedagógicas nas escolas em que atuam. Este trabalho tem como objetivo analisar as contribuições do GMF para a formação docente para a EPT, especialmente pelas Escolas Cidadãs. Para tanto, foram analisados os relatórios e outros instrumentos elaborados pela coordenação do Programa a partir de seminários e atividades de acompanhamento do GMF, aplicadas aos seus participantes durante a capacitação na Finlândia e durante o desenvolvimento dos projetos aplicados nas suas escolas de origem no estado. $A$ análise teve seu foco no perfil de competências e objetivos identificados no desenvolvimento do Programa, a partir de categorias relacionadas a: realidade Brasil x Finlândia e possibilidades de aprimoramento da Educação Brasileira; processo de ensino-aprendizagem sob a perspectiva da abordagem centrada no estudante; metodologias ativas e ferramentas digitais; práticas curriculares diferenciadas; vínculos com a comunidade e outras escolas da Paraíba. Conclui-se que o perfil das competências desenvolvidas no GMF e seus objetivos de aprendizagem relacionados permitem melhorias individuais e coletivas do processo de ensino-aprendizagem, em especial para as Escolas Cidadãs, considerando uma formação direcionada ao protagonismo juvenil.

ABSTRACT. Gira Mundo Finland (GMF) is a Paraíba Government action through the State Secretary Education Office and aims at training career state civil servants at Finnish Universities of Applied Sciences. Both career teachers and managers spend between four to eight weeks immersed in Finland while they visit schools, organizations and experience the Finnish educational system. Upon their return to Paraiba, they develop a project to improve their educational environment in the schools they work. This paper analyses how GMF contributes to teacher training at the vocational teaching level, with special regards to the Citizen Schools. Thus, reports and tools designed by the Program's coordination have been analyzed along with follow-up activities that were carried out with the participants during their stay in Finland and reports that were submitted after their development work projects were finalized. The analysis focused on tracing a competence and aims profile identified within the Program from categories related to: the reality in Brazil vs Finland and the possibilities of improvement in the Brazilian Education; teaching-learning processes under the student-centered approach; active methodologies and digital tools; innovative curricular practices; connections with the community and other schools in Paraíba. We concluded that the profile of the competencies developed in the GMF and its related 
learning objectives allow individual and collective teaching-learning processual improvements, especially for the Citizen Schools, considering teacher training focused on the youth protagonism.

\section{INTRODUÇÃO}

No ano de 2016, o governo da Paraíba implantou duas ações importantes para a melhoria da Educação Profissional no estado: as Escolas Cidadãs (criadas em 2015 pelos Decretos № 36.408 e 36.409, de 30 de novembro de 2015) e o Programa Gira Mundo Finlândia (GMF), que tem promovido a capacitação in loco de professores do estado em Universidades de Ciências Aplicadas finlandesas.

A ampliação das Escolas Cidadãs - em número de oito em 2016, 33 em 2017 e uma projeção de 100 escolas para 2018, atrelada à formação dos profissionais que já atuam ou atuarão nessas escolas, constitui um marco importante na educação do estado da Paraíba, especialmente porque tais escolas apresentam princípios e diretrizes que conduzem ao fortalecimento do protagonismo juvenil e da formação profissional.

Este trabalho tem o propósito de analisar as contribuições do Gira Mundo Finlândia, um dos programas de formação dos profissionais atuantes nas Escolas Cidadãs, para a formação docente demandada para a Educação Profissional e Tecnológica ofertada por esse tipo singular de escola no estado da Paraíba.

\section{O PROGRAMA GIRA MUNDO FINLÂNDIA}

O programa Gira Mundo Finlândia (GMF) é uma iniciativa do Governo do Estado da Paraíba, por meio da Secretaria de Estado da Educação (SEE), e tem como objetivo a capacitação de professores efetivos da Rede Estadual de Ensino da Paraíba em Universidades de Ciências Aplicadas da Finlândia, em parceria com a Fundação de Apoio à Pesquisa do Estado da Paraíba (FAPESQ). O programa teve início em 2016 com o lançamento do Edital de Bolsas № 002/2016 FAPESQ, para a seleção e o envio de vinte professores à Häme University of Applied Sciences - HAMK, na cidade de Hämeenlinna, Finlândia, por um período de 8 semanas. No ano de 2017, o GMF foi ampliado para um total de 55 vagas, das quais 40 foram destinadas à HAMK (Edital de Bolsas ํo 003/2017) e quinze para a Tampere University of Applied Sciences - TAMK (Edital de Bolsas № 004/2017), uma nova universidade parceira que recebeu também gestores das escolas. O programa tem duração de oito semanas na HAMK e quatro semanas na TAMK.

Durante o período de imersão na Finlândia, os participantes têm acesso a um programa de desenvolvimento profissional focado em visitas a escolas e empresas e em experiências em torno de competências profissionais e do aprendizado centrado no estudante. Dentre os temas centrais desenvolvidos no GMF, estão: Empreendedorismo, 
Educação para o Século XXI, Aprendizado baseado em Projetos e Problemas (PBL), Ferramentas Digitais na Educação, Educação Profissional (VET) e Educação Básica. O GMF, portanto, propicia aos participantes desde conhecimentos pedagógicos até a identificação de aspectos a serem aprimorados na sua prática para a melhoria do ensino-aprendizagem e soluções para problemas enfrentados na escola. Representa uma ação inovadora e um investimento alinhado com as políticas educacionais da Paraíba, em direção à melhoria da qualidade da Educação no estado e do bem-estar social nas comunidades atendidas pelas escolas nas três macrorregiões contempladas: Zona da Mata, Agreste e Sertão.

As informações básicas do programa GMF e a previsão de vagas e temas de desenvolvimento de projetos para o ano de 2018 são apresentadas na Tabela 1. Ressalta-se que o Programa GMF apresenta características únicas no cenário nacional por ser um programa de capacitação no qual os participantes desenvolvem um projeto de melhoria das condições de suas escolas após o período de imersão no Sistema Educacional Finlandês, visando o envolvimento de estudantes, de professores, de gestores e da comunidade da área de influência da escola. Por meio desse projeto pretende-se desenvolver uma nova postura identitária docente, a de multiplicadores e agentes de mudança, consolidada por um contexto de superação em que a colaboratividade sustenta e fomenta a formação de comunidades profissionais de aprendizagem.

Quadro 1. Informações básicas do programa Gira Mundo Finlândia nos anos de 2016 e 2017.

\begin{tabular}{|c|c|c|c|}
\hline Ano & Universidade & $\begin{array}{l}\text { № de } \\
\text { vagas }\end{array}$ & Temas do projeto \\
\hline 2016 & HAMK & 20 & $\begin{array}{c}\text { Educação Profissional, Empreendedorismo, PBL e Ferramentas } \\
\text { Digitais na Educação }\end{array}$ \\
\hline 2017 & HAMK & 40 & $\begin{array}{c}\text { Educação Profissional, Educação Básica, PBL e Ferramentas } \\
\text { Digitais na Educação }\end{array}$ \\
\hline 2017 & TAMK & 15 & Empreendedorismo \\
\hline 2018 & HAMK & $40^{*}$ & $\begin{array}{c}\text { Educação Profissional, Educação Básica, PBL e Ferramentas } \\
\text { Digitais na Educação }\end{array}$ \\
\hline 2018 & TAMK & $40^{*}$ & Empreendedorismo, Competências do Século XXI e Inovação \\
\hline
\end{tabular}

OBS.: PBL - Aprendizagem Baseada em Projetos, Problemas e Fenômenos; *: previsão de vagas.

Fonte: os Autores.

\section{AS ESCOLAS CIDADÃS INTEGRAIS NO ESTADO DA PARAÍBA: FORMAÇÃO PROFISSIONAL EM DIREÇÃO AO PROTAGONISMO JUVENIL.}

As Escolas Cidadãs fazem parte das políticas educacionais do Governo da Paraíba, e foram concebidas a partir do comprometimento com a continuação dos estudos dos egressos 
da Educação Básica e com a sua inserção no mundo do trabalho. Existem atualmente duas modalidades de Escolas Cidadãs no Estado da Paraíba: a Escola Cidadã Integral e a Escola Cidadã Integral Técnica. A Escola Cidadã Integral (ECI) foi criada por meio do Decreto № 36.408 de 30 de novembro de 2015, (PARAÍBA, 2015a) e as Escolas Cidadãs Integrais Técnicas (ECIT) por meio do Decreto № 36.409 de 30 de novembro de 2015 (PARAÍBA, 2015b), ambos publicados no Diário Oficial do Estado de 1ํ de dezembro de 2015. O enfoque pedagógico das duas modalidades de Escola Cidadã leva em conta o protagonismo do estudante e a sua consciência no exercício da cidadania, com a diferença do enfoque profissionalizante da ECIT (Art 5 inciso I do Decreto № 36.408/2015 e Art. 5ํinciso I do Decreto № 36.409/2015).

Ambas as modalidades preveem uma jornada de trabalho com carga horária multidisciplinar para os professores, um plano de ação da escola como instrumento de gestão escolar (contendo metas, indicadores e estratégias) que tem como diretriz o plano de ação das Escolas Cidadãs, um programa de ação da equipe escolar com foco na operacionalização dos meios e processos, que tem como referência o plano de ação da escola, e o "Projeto de Vida" dos estudantes, uma documentação contendo suas ambições para o futuro e desenvolvido durante o seu período de estudos nas Escolas Cidadãs (Decretos № 36.408 e 36.409 , de 30 de novembro de 2015). O tempo de permanência dos estudantes nas Escolas Cidadãs (ficam na escola de $7 \mathrm{~h} 30$ às $17 \mathrm{~h} 00$ ) permite que desenvolvam diversas atividades num currículo diversificado e flexível, com destaque para o supracitado Projeto de Vida, que orienta os estudantes a desenvolverem habilidades e competências aproveitando os diversos espaços da escola, como laboratórios específicos e salas temáticas.

\section{DO SISTEMA EDUCACIONAL FINLANDÊS À EDUCAÇÃO PROFISSIONAL BRASILEIRA.}

Conforme apontado pelo Ministério das Relações Exteriores no Brasil (ITAMARATY, 2016) e reforçado por Cotrim-Guimarães e Ouverney-King (2017), a Finlândia tem se destacado pelos seus resultados de excelência não apenas no âmbito educacional, mas em diversos outros indicadores. O Sistema Educacional Finlandês, no que se refere ao nível médio da Educação Básica, possibilita aos estudantes cursar um Ensino Médio regular (General Upper Secondary School) e a Educação Profissional Técnica de nível médio (Vocational Upper Secondary School), esta última, principalmente, por meio da formação profissional integrada ao Ensino Médio, apresentando elementos e organização bastante semelhantes ao modelo brasileiro (COTRIM-GUIMARÃES; OUVERNEY-KING, 2017).

A formação possibilitada aos professores participantes do Gira Mundo Finlândia promoveu, além da aprendizagem de metodologias ativas e ferramentas inovadoras, a integração com escolas, organizações, professores e estudantes finlandeses. Assim, os 
participantes do GMF tiveram a oportunidade de conhecer não apenas aspectos educacionais, mas também sociais, culturais e políticos do país, de forma a reconhecer os elementos que conferem excelência ao Sistema de Ensino Finlandês, ao mesmo tempo em que foram capazes de comparar e identificar possibilidades de aplicação e melhoria no Sistema Educacional Brasileiro, de modo geral, e no Sistema Educacional Paraibano, de modo particular, sem desconsiderar, para tanto, as dimensões culturais, políticas, sociais e econômicas brasileiras.

Esse contato direto com a realidade educacional finlandesa, e ainda a aprendizagem efetiva dessas novas ferramentas e metodologias ativas, se deu por meio de atividades em que os professores participantes do Programa atuam como protagonistas do processo de aprendizagem (student centered learning - aprendizagem centrada no estudante) e o processo de aprendizagem se efetiva de forma que o estudante aprende enquanto pratica (learning by doing - aprender fazendo). Sendo assim, o processo de ensino-aprendizagem inerente ao Programa Gira Mundo Finlândia permitiu aos seus participantes não apenas a apropriação dos conhecimentos e habilidades, mas também a identificação de aspectos e objetivos importantes a serem aprimorados na sua prática docente, quando retornam à docência no Brasil.

Tais conhecimentos e habilidades estão intrinsecamente relacionados à Educação Profissional, especialmente à ofertada pelas Escolas Cidadãs, já que o próprio Sistema Educacional Finlandês apresenta, no seu cerne, uma formação integrada, com aspectos bastante semelhantes às discussões e propostas de currículo integrado para a Educação Profissional de nível médio no Brasil (BARBOSA et al., 2016; COTRIM-GUIMARÃES; OUVERNEY-KING, 2017), formação esta proposta para as Escolas Cidadãs Integrais e Escolas Cidadãs Integrais Técnicas na Paraíba.

Segundo Sahlberg (2015), o Sistema Educacional Finlandês tem o seu foco no desenvolvimento de habilidades sociais, empatia e liderança, dedicando-se de forma considerável ao desenvolvimento de atividades como projetos integrados, oficinas e outras ações que levem em conta as demandas individuais e comunitárias e os planos individuais dos estudantes. O reconhecimento dessas características, a aprendizagem de novas metodologias, técnicas e ferramentas e outras reflexões propiciadas pelo Gira Mundo Finlândia, portanto, vão ao encontro da proposta de formação das Escolas Cidadãs, que por sua vez têm como base de sustentação a formação de "jovens autônomos, solidários e competentes atores e sujeitos da própria ação e prontos a buscar a solução de problemas reais na escola, na comunidade e na vida social mais ampla" (SEE-PB, 2016, p.03). Além disso, se propõem à formação de sujeitos ativos, capazes de tomar decisões que considerem não apenas sua individualidade, mas também o coletivo (lbid), o que remete a uma questão importante da Educação Profissional no Brasil: a necessidade de superação de uma formação centrada nos interesses do mercado, em direção a uma formação que tenha sua centralidade 
nos interesses dos estudantes, como sujeito de necessidades, de desejos e potencialidades (RAMOS, 2008), o que não exclui a formação dos estudantes para ingresso no mercado. Nesse ponto, é importante registrar alguns aspectos presentes na Educação Profissional Brasileira. Segundo Lüscher e Dore (2011, p.154), "a marca distintiva da escola média no Brasil, desde a década de 1930 do século XX até hoje, é a dualidade de seus objetivos e de sua organização. De um lado, está a formação geral, tendo em vista a continuidade de estudos no nível superior; de outro, a formação profissional, com foco mais imediato no mercado de trabalho".

Ramos (2017) corrobora com essa afirmação, quando aponta que o Ensino Médio no Brasil sempre teve sua razão de ser vinculada ao mercado de trabalho, seja ele integrado à Educação Profissional ou não. Esse vínculo se dá tanto pela possibilidade imediata de inserção no mercado de trabalho após conclusão desse nível de ensino, como pela forma mediata, em que o estudante vislumbra o acesso ao curso superior para após sua conclusão ingressar no mercado. Ou seja, como extensão do Ensino Médio, o vestibular e outros processos seletivos também apontam a centralidade do mercado de trabalho no Ensino Médio e Educação Profissional.

Ao se tomar como centralidade os sujeitos (estudantes) e suas necessidades, estes passam a ser considerados não como sujeitos abstratos e isolados, mas como afirma Ramos (2008), "sujeitos singulares cujo projeto de vida se constrói pelas múltiplas relações sociais, na perspectiva da emancipação humana, que só pode ocorrer à medida que os projetos individuais entram em coerência com um projeto social coletivamente construído"(p. 05).

Colocar os estudantes na centralidade do processo de ensino-aprendizagem, repensar e desenvolver novas estratégias de ensino, novas possibilidades de inserção desses jovens no processo educativo, em direção ao protagonismo juvenil, é também uma forma de refletir sobre esses sujeitos que deveriam tornar-se o foco do projeto educacional do Ensino Médio regular e profissional, conforme apontado anteriormente por Ramos (2008): a juventude que compreende o público atendido por esse nível de ensino, que tem sofrido mudanças quanto à sua configuração que não podem ser desconsideradas no projeto formativo em pauta. Afinal, segundo Dayrell (2001, p. 140), "uma outra forma de compreender esses jovens que chegam à escola é apreendê-los como sujeitos sócio-culturais". E ainda, "Trata-se de compreendê-lo na sua diferença, enquanto indivíduo que possui uma historicidade, com visões de mundo, escalas de valores, sentimentos, emoções, desejos, projetos, com lógicas de comportamentos e hábitos que Ihe são próprios" (Ibid).

Assim é que o protagonismo juvenil se insere como pilar das Escolas Cidadãs, na medida em que busca promover a integração da juventude por meio de uma nova forma de participação, descrita por Souza (2006, p. 256) como "inscrição ativa" dos jovens, em que “(...) o jovem torna-se um 'portador ativo' de um discurso que parece emitido por ele. Pode-se afirmar que o apelo a uma posição de protagonismo, justificada pela exaltação de supostas 
qualidades e capacidades juvenis, funcione como estratégia de motivação para a integração e para o estímulo à 'adesão ativa' das novas gerações ao discurso do poder".

$E$ é justamente nesse ponto que estabelecemos a relação entre os conhecimentos e habilidades desenvolvidos pelos professores participantes do Gira Mundo Finlândia e o projeto educativo para as Escolas Cidadãs da Paraíba, focadas no projeto de vida de cada sujeito/estudante e no desenvolvimento do protagonismo juvenil. A análise dos materiais e documentos produzidos pela coordenação do Programa permitiu fazer esses apontamentos, que seguem logo após a explicitação da metodologia utilizada.

\section{METODOLOGIA}

Esse trabalho consiste na análise de relatórios e outros instrumentos elaborados pela coordenação do Programa Gira Mundo Finlândia (GMF), considerando as duas turmas enviadas à Finlândia, em 2016 e 2017. O acompanhamento das atividades, as entrevistas e questionários aplicados pela coordenação aos participantes do Programa tiveram como resultados a elaboração de relatórios, incluindo a construção de um perfil de competências e objetivos organizado a partir das impressões e colocações dos professores participantes sobre o GMF, de modo geral, sobre o desenvolvimento dos projetos nas escolas onde atuam na Paraíba, mas principalmente, sobre o Sistema de Ensino Finlandês e as possibilidades de melhoria da Educação Básica e Profissional no Brasil (e mais especificamente na Paraíba) a partir das reflexões e mudanças na prática docente desses professores em formação.

Essa análise teve como foco o perfil de competências acima referido, que segundo o relatório apresentado pela coordenação do Programa (COTRIM/SEE-PB, 2017) tem o propósito de orientar tanto o desenvolvimento de novas turmas do Programa quanto a direcionar a avaliação do Programa a médio e longo e prazo. Para elaboração desse perfil, as respostas e apontamentos dos professores participantes foram categorizados e organizados de forma a embasar a proposição das competências. Foram identificadas cinco categorias diferentes, mas articuladas e complementares, visto que, no seu conjunto, contribuem para o aprimoramento do processo de ensino-aprendizagem, considerando os pilares da Educação para o Século XXI e fomentando o protagonismo juvenil.

Nesse relatório, considerou-se competência como "a capacidade de mobilizar conhecimentos, habilidades e valores no desenvolvimento de determinadas atividades, sem desconsiderar o contexto em que estão inseridas" (COTRIM/SEE-PB, 2017, s/p). Sendo assim, dada a amplitude de cada competência, foram identificados seus respectivos objetivos que, por sua vez, direcionam as ações que permitem inferir o seu desenvolvimento.

Apresenta-se a seguir a análise dos documentos, especialmente o relatório técnico com o perfil de competências e objetivos (COTRIM/SEE-PB, 2017), vez que este documento 
integra e consolida o conjunto de conhecimentos e habilidades desenvolvidas pelos participantes e suas possibilidades para melhoria da educação na Paraíba.

\section{COMPETÊNCIAS E OBJETIVOS DO PROGRAMA GMF: CONTRIBUIÇÕES DA EDUCAÇÃO FINLANDESA PARA A EDUCAÇÃO PROFISSIONAL NA PARAÍBA.}

Conforme indicado no capítulo anterior, cinco categorias foram identificadas e embasaram a construção das competências e seus respectivos objetivos. É importante ressaltar que estes foram elaborados considerando-se a percepção e apontamentos dos professores participantes do Programa GMF. Isso significa que, sendo eles professores já atuantes nas Escolas Cidadãs e em outras escolas convencionais do estado, esses resultados indicam possibilidades e prováveis contribuições da formação desses professores na Finlândia para a implementação do projeto educativo que orienta as Escolas Cidadãs no estado da Paraíba, dada a significativa expansão a partir de 2018.

As cinco categorias identificadas foram reorganizadas sob a forma de temas, compreendendo: (i) Brasil X Finlândia: confrontando realidades para o aprimoramento da educação brasileira; (ii) relação professor-aluno no processo de ensino aprendizagem sob a perspectiva da abordagem centrada no estudante; (iii) metodologias ativas e ferramentas digitais; (iv) práticas curriculares diferenciadas e (v) fortalecendo vínculos com a comunidade e outras escolas da Paraíba. Considerando os temas relacionados à formação e atuação dos professores participantes do GMF, foram identificadas as seguintes competências que deverão nortear o desenvolvimento e avaliação do Programa (COTRIM/SEE-PB, 2017):

- Definir e aplicar estratégias individuais e coletivas para o enfrentamento da realidade brasileira, espelhando-se nos aspectos da sociedade e do Sistema Educacional Finlandeses que podem contribuir para se repensar e redefinir práticas educacionais no Brasil

- Promover um processo de ensino-aprendizagem que possibilite aos estudantes maior protagonismo e corresponsabilidade no desenvolvimento das atividades pedagógicas e na sua relação com o professor.

- Aprimorar sua prática docente por meio da adoção de novas metodologias e ferramentas de ensino-aprendizagem que estimulem o trabalho colaborativo e o desenvolvimento da autonomia e resolutividade dos estudantes.

- Implementar novas práticas curriculares individuais e coletivas, contribuindo, inclusive, para incrementar as reflexões e definições sobre currículo na(s) escola(s) onde atua.

- Fortalecer o vínculo com a comunidade atendida pela escola e com a rede de escolas do estado da Paraíba, de forma a consolidar a identidade da instituição. 
Não se pretende, aqui, registrar os objetivos relacionados à cada competência identificada, mas discutir como eles estabelecem uma relação com o projeto formativo das Escolas Cidadãs e com um projeto de Educação profissional cuja centralidade esteja nos sujeitos.

Verificou-se que a formação propiciada pelo Programa GMF possibilitou aos professores uma reflexão sobre a Educação Brasileira como um todo e mais especificamente sobre a educação na Paraíba, a partir de uma comparação entre a realidade finlandesa e a brasileira. Embora muitos professores tenham apontado problemas e obstáculos à implantação de modelos inovadores no Brasil, essa comparação tem permitido uma apropriação de elementos do Sistema Educacional Finlandês que podem contribuir para se repensar e redefinir práticas educacionais no Brasil, bem como "direcionar e contribuir para a definição e implementação de melhorias na infraestrutura e ambiente escolar, de forma a propiciar o aprimoramento do processo de ensino-aprendizagem e a implementação de novas práticas pedagógicas" (COTRIM/SEE-PB, 2017, s/p).

Sobre essa questão, Cotrim-Guimarães e Ouverney-King (2017) apontam algumas características do Sistema Educacional Finlandês que, integrados à formação docente para a EPT, podem contribuir para uma formação profissional que tenha sua centralidade nos sujeitos: aprendizagem centrada no estudante, desenvolvimento de habilidades sociais, autonomia e cooperação, além de possibilidades de mudança no desenho curricular (que, aliás, consiste numa das categorias relacionadas aos apontamentos dos participantes do GMF). Essas mesmas características foram ressaltadas por Ryymin, Kunnari e D’Andrea (2017) em considerações a respeito de um programa de capacitação no Sistema Finlandês realizado pela Rede Federal de Educação Profissional, Científica e Tecnológica, atendendo a participantes de diversas áreas do conhecimento e adotando uma abordagem multidisciplinar e aplicada diretamente à formação docente para EPT, com ênfase em inovações na educação e competências profissionais desenvolvidas a partir de trabalhos colaborativos.

No caso do Gira Mundo Finlândia no estado da Paraíba, os participantes indicam ainda a importância de se compartilhar a experiência na Finlândia e envolver os professores que não tiveram a oportunidade de participar do Programa nas ações de divulgação, aprendizagem de novas práticas e estratégias de ensino, sempre em direção à definição e implementação de melhorias individuais e coletivas do processo de ensino-aprendizagem.

Nessa direção, ressalta-se a temática sobre o fortalecimento dos vínculos com a comunidade e outras escolas da Paraíba, o que configura, de certa forma, estabelecer um trabalho em rede entre as escolas do estado, ainda que esse seja apenas o pontapé inicial para o fortalecimento da rede de colaboração entre as escolas do estado.

Esse direcionamento é muito importante, primeiro pela própria importância do envolvimento da comunidade e familiares e atendimento às suas demandas; segundo, pela possibilidade de troca de experiências entre os profissionais das escolas, e não menos 
importante, porque o estreitamento desse vínculo entre escolas, e entre escola e comunidade, contribui para a formação dos próprios estudantes, sempre em direção ao protagonismo juvenil.

E por falar em protagonismo juvenil, uma outra temática registrada refere-se à relação professor-aluno no processo de ensino-aprendizagem sob a perspectiva da abordagem centrada no estudante. Os professores participantes do GMF apontaram características bastante presentes no Sistema Educacional Finlandês, como um todo, que podem ser apropriadas e adequadas à realidade de brasileira, de forma a possibilitar aos estudantes maior protagonismo e corresponsabilidade no desenvolvimento das atividades pedagógicas e na sua relação com o professor.

Os objetivos elencados para essa categoria e sua competência orientam desde considerar os interesses e participação dos estudantes no planejamento e outras definições do processo de ensino-aprendizagem, passando pela implementação de trabalho colaborativo e outras atividades coletivas; aplicação de ferramentas e estratégias que estimulem a criatividade, autonomia e confiança; até estimular os jovens a participar de processos decisórios, colegiados, entidades estudantis e outras ações coletivas no âmbito escolar.

Os professores participantes também indicaram como forma de aprimorar a prática docente a adoção de novas metodologias e ferramentas de ensino-aprendizagem, o que consistiu numa outra categoria identificada no relatório. Dessa forma, pretende-se fomentar o trabalho colaborativo e o desenvolvimento da autonomia e resolutividade dos estudantes. No contexto de programas de capacitação baseados no Sistema Educacional Finlandês, Ryymin, Kunnari e D'Andrea (2017) ressaltam a importância da busca por soluções digitais e do uso de ferramentas digitais de maneira criativa e colaborativa, a fim de promover um impacto positivo na atuação dos professores por meio da melhoria do seu bem-estar e da adoção de uma postura colaborativa, incluindo o suporte a seus pares no ambiente de trabalho. Os autores verificaram a melhoria da autoestima dos participantes na consolidação de redes de relacionamento profissionais com a aplicação criativa de soluções digitais na facilitação dos processos envolvendo o trabalho colaborativo. Como consequência, a adoção dessas novas práticas no processo de ensino e aprendizagem requer um ajustamento do processo avaliativo. Nessa direção, a diversificação das atividades avaliativas, incluindo aquelas adequadas às metodologias ativas e colaborativas de aprendizagem, é uma importante estratégia para estimular a confiança e responsabilidade dos estudantes em relação aos estudos (COTRIM/SEE-PB, 2017).

Por fim, em relação às práticas curriculares, propõe-se a implementação de novas práticas individuais e coletivas, contemplando desde a implementação de metodologias ativas no desenvolvimento dos conteúdos, articulação entre docentes e integração entre estes e seus conteúdos e adoção de abordagens interdisciplinares. Com isso, pretende-se possibilitar e/ou aprimorar a problematização, integração e contextualização dos conteúdos. 
Nesse ponto, evidencia-se a noção de currículo como o definido por Moreira (2001, p. 64): “(...) poderíamos pensar currículo se referindo às experiências que giram em torno do conhecimento que nós organizamos e desenvolvemos junto aos nossos estudantes". E essa definição, atrelada à discussão de Simões (2007, apud Ramos, 2008) a seguir, demonstra como as categorias, temas, competências e objetivos construídos pelo/para Programa Gira Mundo Finlândia se constituem num todo integrado e articulado, possibilitando aos profissionais em formação, atuantes ou não na EPT, implementar uma educação focada nos sujeitos humanos.

O ensino técnico articulado com o ensino médio, preferencialmente integrado, representa para a juventude uma possibilidade que não só colabora na sua questão da sobrevivência econômica e inserção social, como também uma proposta educacional, que na integração de campos do saber, torna-se fundamental para os jovens na perspectiva de seu desenvolvimento pessoal e na transformação da realidade social que está inserido" (SIMÕES, 2007, apud RAMOS, 2008, s/p).

\section{CONSIDERAÇÕES FINAIS}

Diante dos resultados apresentados e da análise dos documentos relacionados ao programa Gira Mundo Finlândia (GMF), considerando as experiências vivenciadas pelos participantes na Finlândia e a aplicação do projeto de desenvolvimento local nas suas escolas de origem no retorno à Paraíba, conclui-se que o perfil das competências desenvolvidas e seus objetivos de aprendizagem relacionados permitem melhorias individuais e coletivas do processo de ensino-aprendizagem.

A experiência na Finlândia tem possibilitado aos professores da Paraíba o acesso a novas metodologias, ferramentas e práticas que consideram os estudantes sujeitos ativos e protagonistas do processo de ensino-aprendizagem, o que, por sua vez, vai ao encontro dos princípios e pilares de sustentação das Escolas Cidadãs, ressaltando o protagonismo juvenil e uma Educação Profissional centrada nos interesses dos estudantes. Além disso, a identificação das competências profissionais desenvolvidas pelos participantes do Programa representa uma importante ferramenta para o acompanhamento, alinhamento e monitoramento da qualidade do GMF, além de permitir a geração de índices comparativos para a análise dos resultados efetivos alcançados pelo Programa.

\section{REFERÊNCIAS}

BARBOSA et al. Rethinking the curriculum: calling teachers to discuss and propose new perspectives for the curricular design. Tampere - FI: Tamk, 2016. [Relatório técnico] COTRIM, I.M.A. (SECRETARIA DE ESTADO DA EDUCAÇÃO DA PARAÍBA - SEE/PB). Perfil de competências e objetivos do Programa da Secretaria de Estado da Educação da Paraíba: "Gira Mundo" - Finlândia. João Pessoa, PB, 2017. [Relatório técnico] COTRIM-GUIMARÃES, I.M.A. OUVERNEY-KING, J.R. Por dentro do Sistema Educacional Finlandês: elementos para se repensar o ensino médio integrado no Brasil. In: ARAÚJO, A.C. 
SILVA, C.N.N (Orgs.) Ensino Médio Integrado no Brasil: fundamentos, práticas e desafios. Brasília: DF, Ed. IFB, 2017, p.54-70.

DAYRELL, Juarez. A escola como espaço sócio-cultural. In: Múltiplos olhares sobre educação e cultura. $2^{\text {a }}$ reimpressão. Belo Horizonte: Editora UFMG, 2001.

LÜSCHER, Ana Zuleima; DORE, Rosemary. Política Educacional no Brasil: educação técnica e abandono escolar. Revista Brasileira de Pós Graduação, Brasília, supl.1, v. 8, p.147-176, dez. 2011.

MINISTÉRIO DAS RELAÇÕES EXTERIORES (ITAMARATY). O sistema de ensino finlandês: os pilares de uma sociedade baseada no conhecimento. In:

Mundo Afora: Educação Básica e Ensino Médio. Disponível em: <http://www.dc.itamaraty.gov.br/publicacoes/colecao-mundo-afora/Mundo\%20Afora\%20n11\%20v11\%20WEB\%20single.pdf/ $\geq$ Acesso em: 30 set. 2016.

MOREIRA, Antônio Flávio Barbosa. O currículo da graduação hoje: possibilidades e desafios. In: UNIMONTES, Anais I Forum de Graduação da Unimontes / II Fórum de Graduação da Unimontes. Montes Claros (MG): Ed. Unimontes, 2001, p. 61-71.

PARAÍBA, Governo do Estado. Diário Oficial do Estado da Paraíba. Decreto № 36.408 de 30 de Novembro de 2015. Cria a Escola Cidadã Integral, institui o Regime de Dedicação Docente Integral - RDDI e dá outras providências. Diário Oficial do Estado № 15.994 de 01 de Dezembro de 2015. Pág. 1-2. 2015a. Disponível em < http://static.paraiba.pb.gov.br/2015/12/Diario-Oficial-01-12-2015.pdf>. Acesso em: 29 dez. 2017.

PARAÍBA, Governo do Estado. Diário Oficial do Estado da Paraíba. Decreto № $\mathbf{3 6 . 4 0 9}$ de $\mathbf{3 0}$ de Novembro de 2015. Cria a Escola Cidadã Integral Técnica, institui o Regime de Dedicação Docente Integral - RDDI e dá outras providências. Diário Oficial do Estado № 15.994 de 01 de Dezembro de 2015. Pág. 2-3. 2015b. Disponível em < http://static.paraiba.pb.gov.br/2015/12/Diario-Oficial-01-12-2015.pdf>. Acesso em: $29 \mathrm{dez}$. 2017.

RAMOS, M.. Concepção do Ensino Médio Integrado. [2008] Disponível em http://forumeja.org.br/go/sites/forumeja.org.br.go/files/concepcao_do_ensino_medio_integrado5.pdf Acesso em 23 jun 2017.

RYMMIN, E.; KUNNARI, I.; D'ANDREA, A.F. Digital Solutions in Teacher Education enhance Wellbeing and Expertise. AMK-LEHTI/UAS JOURNAL. Journal of Finnish Universities of Applied Sciences. 2017. Disponível em < https://uasjournal.fi/koulutus-oppiminen/digital-solutions-in-teacher-education-enhance-wellbeing-and-expertise/\#1458134585005b3f22396-5506>. Aceso em: 29 dez. 2017.

SAHLBERG, P. Finnish Lessons: What can the world learn from educational change in Finland? 2 ed. New York (USA): Teachers College / Columbia University, 2015.

SECRETARIA DE ESTADO DA EDUCAÇÃO DA PARAÍBA - SEE-PB. Diretrizes para o funcionamento das Escolas Cidadãs Integrais e Escolas Cidadãs Integrais Técnicas. João Pessoa: PB, 2016. Disponível em < http://paraiba.pb.gov.br/downloads/DIRETRIZES 2018 PARA DIVULGA\%C3\%87\%C3\%830.pdf >. Acesso em: 28 de dezembro de 2017.

SOUZA, R.M. O Discurso do Protagonismo Juvenil. São Paulo, 2006. 351 f. Tese (Doutorado em Sociologia) - Faculdade de Filosofia, Letras e Ciências Humanas, Universidade de São Paulo. 